\title{
Review: bright light therapy and dawn simulation reduce symptom severity in seasonal affective disorder
}

Golden RN, Gaynes BN, Ekstrom RD, et al. The efficacy of light therapy in the treatment of mood disorders: a review and metaanalysis of the evidence. Am J Psychiatry 2005; 162:656-62.

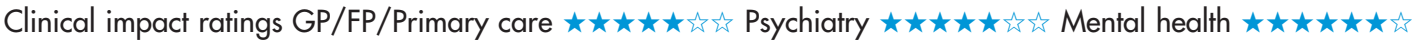

\section{Is light therapy efficacious for treatment of mood disorders?}

\section{METHODS}

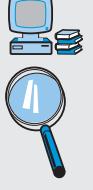

Data sources: Medline (1975 to July 2003), Cochrane Library and bibliographies of relevant reviews and studies.

Study selection and assessment: English language randomised controlled trials (RCTs) of adults 18-65 years of age who had a diagnosis of mood disorder based on DSM-III, DSM-III-R, DSM$I V$, Research Diagnostic Criteria, or the Rosenthal criteria and were in the acute phase of treatment. Treatment conditions had to meet the following minimum dose criteria: bright light therapy for seasonal affective disorder ( $\geqslant 4 \mathrm{~d}$ of $\geqslant 3000$ lux-h) $v$ placebo ( $\leqslant 300$ lux); dawn simulation (increasing light exposure from 0 to 200-300 lux over $1-2.5$ h) vs placebo $(<5$ lux increase or $<15$ min duration); and bright light augmentation ( $\geqslant 4 \mathrm{~d}$ of $\geqslant 3000$ lux-h and bright light therapy as the primary adjunct to standard therapy).

Outcomes: psychiatric symptom measures (eg, Hamilton Depression Rating scale, Seasonal Affective Disorders Version).

\section{MAIN RESULTS}

20 RCTs $(n=693)$ met the inclusion criteria and had sufficient data to calculate mean scores and standard deviations. Meta-analyses showed that bright light therapy and dawn simulation reduced depressive symptom severity more than placebo for seasonal affective disorder (table). Bright light therapy also reduced symptom severity more than placebo for non-seasonal depression (table) but not when used as an adjunct to pharmacotherapy. Analysis of 4 trials that included data on remission (ie, scores $\leqslant 8$ on Hamilton Depression Rating Scale) showed increased remissions with bright light therapy in patients with seasonal affective disorder (odds ratio $2.9,95 \%$ CI 1.6 to 5.4 )

\section{CONCLUSIONS}

Bright light therapy reduces the severity of depressive symptoms more than placebo for seasonal affective disorder and non-seasonal depression. Dawn simulation reduces symptom severity for seasonal

For correspondence: Dr R N Golden, University of North Carolina at Chape Hill, Chapel Hill, NC, USA. robert_golden@med.unc.edu

Source of funding: no external funding. affective disorder. Bright light therapy as an adjunct to standard pharmacotherapy does not differ from placebo for non-seasonal depression.

Abstract and commentary also appear in ACP Journal Club.

\section{Commentary}

M otivated by the slow dissemination of light therapy into everyday practice, Golden et al systematically reviewed evidence from randomised trials of the efficacy of light therapy for seasonal and non-seasonal depression. They found consistent evidence of the efficacy of both bright light therapy and dawn simulation for seasonal depression and of bright light therapy for non-seasonal depression.

Some limitations should be mentioned. Studies were modest in size, and the database was probably not large enough to assess the possibility of publication bias. All studies of dawn simulation were conducted by a single research group.

For patients with seasonal depression, bright light therapy land possibly dawn simulation) can be considered safe and effective. Practitioners need to consider the prevalence of such patients in their practices and whether they should be doing anything different to identify them. Most large community and primary care surveys have not assessed seasonal depression, so a comprehensive picture is not available. Several small surveys report prevalence rates of $1-9 \%{ }^{1}$

The findings of the meta-analysis by Golden et al raise the question of whether bright light therapy should be offered as a first line treatment for patients with non-seasonal depression. The available evidence (based on 127 patients in 3 trials) does not yet support firm conclusions on efficacy, and direct comparisons with standard first line treatment (antidepressant medication or structured psychotherapy) are lacking. It seems likely, however, that a substantial number of patients might find light therapy more acceptable than current alternatives. Direct comparisons of both efficacy and effectiveness seem necessary.

Gregory Simon, MD, MPH Center for Health Studies, Group Health Cooperative Seattle, Washington, USA

1 Magnusson A. An overview of epidemiological studies on seasonal affective disorder. Acta Psychiatr Scand 2000;101:176-84.

Bright light therapy $v$ placebo for mood disorders at 6-42 days ${ }^{*}$

\begin{tabular}{llcc}
\hline Therapy & Diagnosis & Number of trials $(\mathbf{n})$ & Standardised mean difference (95\% Cl) \\
\hline Bright light & Seasonal affective disorder & $8(360)$ & $0.84(0.60$ to 1.08$)$ \\
& Non-seasonal depression & $3(127)$ & $0.53(0.18$ to 0.89$)$ \\
Adjunctive bright light & Non-seasonal depression & $5(135)$ & $-0.01(-0.36$ to 0.34$)$ \\
Dawn simulation & Seasonal affective & $5(133)$ & $0.73(0.37$ to 1.08$)$ \\
\hline
\end{tabular}

${ }^{*} \mathrm{Cl}$ defined in glossary. Differences $>0$ favour treatment. 